\title{
An algorithm for localization of cylinders by reflection mode scattered field
}

\author{
A. Brancaccio, C. Di Dio, and G. Leone \\ Dipartimento di Ingegneria dell'Informazione - Seconda Universita' di Napoli, Italy
}

Received: 27 June 2008 - Revised: 11 September 2008 - Accepted: 24 September 2008 - Published: 14 November 2008

\begin{abstract}
The problem of localizing perfectly electrical conducting cylinders of arbitrary radius is dealt with in a two dimensional geometry. A reflection mode multi-frequency multi-bistatic configuration is considered, and a linear inversion algorithm based on the truncated singular values decomposition is proposed. The algorithm is validated against simulated data, and the effect of errors in the knowledge of the cylinders' radius is discussed.
\end{abstract}

\section{Introduction}

The reflection mode configuration is used, in non destructive testing and subsurface prospecting, in every circumstance where the investigation domain is accessible from only one side. For instance, this happens in the detection and localization of buried pipes or in NDT of rebars in concrete, and in general in ground penetrating radar applications. In this paper the problem of localizing objects by measurements of the electromagnetic field scattered when observed in reflection mode is considered for Perfectly Electrical Conducting (PEC) cylinders.

The localization problem has been already addressed in the hypothesis of "small" (with respect to the wavelength) scatterers (Pierri et al., 2005, and references therein). Otherwise, a "shape" reconstruction inverse problem can be formulated (Soldovieri et al., 2005, and references therein).

Here, we propose an algorithm for the localization of PEC circular cylinders whose radius can be comparable or larger than the wavelength. The algorithm is based on the inversion of a linear operator, strictly related to the one used in (Pierri et al., 2005). The fact that the scatterers are not "thin" results in the presence of a frequency dependent factor outside the

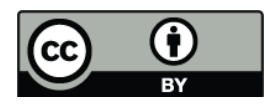

Correspondence to: A. Brancaccio (adriana.brancaccio@unina2.it) integral operator linking data and unknown. Such a factor depends on the shape of the scatterer section, that is a circle in the present case. If the cylinder's radius is not known an error in localization arises and how it affects the reconstructed image is also discussed.

The paper is organized as follows. In Sect. 2 the problem is formulated and the inversion algorithm is introduced. In Sect. 3 the dependence of the inversion result on the correct knowledge of the cylinders' radius is discussed. In Sect. 4 a numerical analysis is performed and results obtained by simulated data are shown. Conclusions follow.

\section{Formulation of the problem}

Let us consider $N$ infinitely long circular PEC cylinders illuminated by TM polarized plane waves. We want to find the positions of the centers of the cylinders, that are assumed to be embedded within a homogeneous medium of known permittivity $\epsilon_{r}$ and residing within the investigation domain $D$. Furthermore, we assume that all the cylinders have the same radius $a$. The problem at hand is a two dimensional one. From now onwards we indicate the centers of the cylinders' circular cross sections as cylinder's positions. The field scattered by the $\mathrm{n}$-th cylinder can be written as (Harrington, 2001)

$E_{s}^{n}=-E_{0} \sum_{p=-\infty}^{+\infty}(-j)^{p} \frac{J_{p}(k a)}{H_{p}^{(2)}(k a)} e^{j p\left(\theta_{n}-\theta_{i}\right)} H_{p}^{(2)}\left(k r_{n}\right)$

where $E_{0}$ is the incident field amplitude at the cylinder position, $k$ is the wavenumber, $\theta_{i}$ is the incidence angle, $J_{p}(\cdot)$ is the Bessel function of order $p, H_{p}^{(2)}(\cdot)$ is the Hankel function of order $p$ and second kind, $\left(r_{n}, \theta_{n}\right)$ denote the polar coordinates of the observation point in the local reference system of the n-th cylinder (see Fig. 1).

Published by Copernicus Publications on behalf of the European Geosciences Union. 


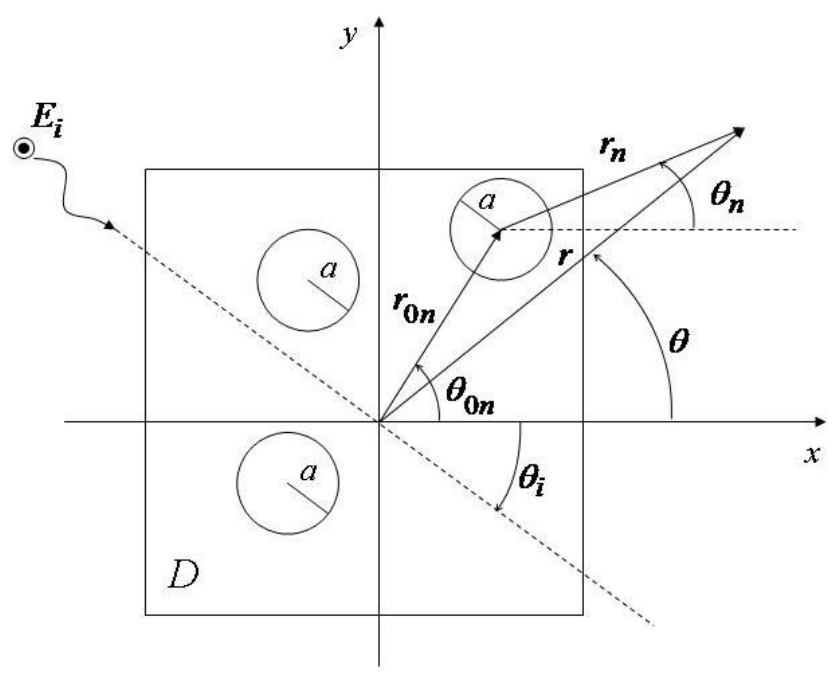

Fig. 1. Geometry of the problem.

The scattered far field, in a gobal reference system $(r, \theta)$ whose origin is located somewhere inside the investigation domain $D$, can be easily derived from Eq. (1) by translating the origin of the system and approximating for large argument the Hankel function. It results:

$$
\begin{aligned}
& E_{s}^{n}=-E_{0} \sqrt{\frac{2 j}{\pi k r}} e^{-j k r} \sum_{p=-\infty}^{+\infty} \frac{J_{p}(k a)}{H_{p}^{(2)}(k a)} e^{j p\left(\theta-\theta_{i}\right)} . \\
& \cdot e^{-j k r_{0 n}\left[\cos \left(\theta_{0 n}-\theta_{i}\right)-\cos \left(\theta_{0 n}-\theta\right)\right]}
\end{aligned}
$$

where $r_{0 n}$ and $\theta_{0 n}$ denote the position of the $\mathrm{n}$-th cylinder and the factor $e^{-j k r_{0 n}\left[\cos \left(\theta_{0 n}-\theta_{i}\right)\right]}$ accounts for the incident field phase at that position. Then, if the mutual scattering between the cylinders is neglected, the field scattered by all the cylinders is given by the superposition $\sum_{n=1}^{N} E_{s}^{n}$ :

$$
\begin{aligned}
& E_{s}=-E_{0} \sqrt{\frac{2 j}{\pi k r}} e^{-j k r} \sum_{p=-\infty}^{+\infty} \frac{J_{p}(k a)}{H_{p}^{(2)}(k a)} e^{j p\left(\theta-\theta_{i}\right)} . \\
& \cdot \sum_{n=1}^{N} e^{-j k r_{0 n}\left[\cos \left(\theta_{0 n}-\theta_{i}\right)-\cos \left(\theta_{0 n}-\theta\right)\right]}
\end{aligned}
$$

Our aim is to determine the position of the scatterers starting from the observed scattered field.

However, it is not convenient to deal with the problem using directly as unknowns the coordinates $r_{0 n}$ and $\theta_{0 n}$, because the relationship between data and unknowns would be strongly non linear. In order to linearize the problem we introduce the auxiliary distributional function (Pierri et al., 2005):

$\gamma(\underline{r})=\sum_{n=1}^{N} \delta\left(\underline{r}-\underline{r}_{0 n}\right)$ where $\underline{r}$ is the position vector and $\underline{r}_{0 n}$ is the vector indicating the position of the $n$-th cylinder in the reference plane.

Now, the problem at hand can be recast as the inversion of the following linear operator equation:

$E_{s}=F(k a) \int_{D} \gamma(x, y) e^{-j(u x+v y)} d x d y$

where

$F(k a)=-E_{0} \sqrt{\frac{2 j}{\pi k r}} e^{-j k r} \sum_{p=-P}^{P} \frac{J_{p}(k a)}{H_{p}^{(2)}(k a)} e^{j p\left(\theta-\theta_{i}\right)}$

and $u=k\left(\cos \theta_{i}-\cos \theta\right), v=k\left(\sin \theta_{i}-\sin \theta\right)$, the cartesian coordinates $x, y$ have been used inside the integral and the sum has been truncated according to the rule $P \approx k a$ (provided $k a \gg 1$ ) (Brancaccio et al., 1998). The function $\gamma(\underline{r})$ is the unknown to be recovered. Its maxima give the searched for positions. Before going on it is worth to make two comments about Eq. (5):

1. the integral operator appearing at the second member is the same studied in (Pierri et al., 2005) for the localization of thin scatterers;

2. the information about the 'size' of the scatterers stands as a frequency dependent factor $F(k a)$.

According to Eqs. (5) and (4), we solve the problem of determining the location of the cylinders from the knowledge of $E_{s}$ in three steps. First, $E_{s}$ is normalized to the factor $F(k a)$. This step requires that the radius $a$ is the same for all the involved scatterers and that it is known. We discuss in the following about such point. Second, the distribution $\gamma$ is approximately reconstructed, which amounts to invert a linear integral operator. This task is accomplished by means of the Singular Value Decomposition (SVD) approach, that allows to regularize the problem by truncating the expansion according to the uncertainties level (TSVD inverse scheme). Third, the total number of scatterers and their unknown positions are determined as the locus of points where the retrieved distribution achieves its maxima. In order to find such points a threshold is introduced below which the reconstruction is discarded. In particular, the threshold is chosen using the results reported in (Liseno et al., 2004).

As far as the first step is concerned, let us point out that the normalization of an operator to a frequency dependent factor could change the singular values behavior, thus affecting the performances of the inversion algorithm in dependence on the threshold level chosen for the SVD truncation (chosen in connection with the signal to noise ratio, ultimately) (Persico and Soldovieri, 2004). 


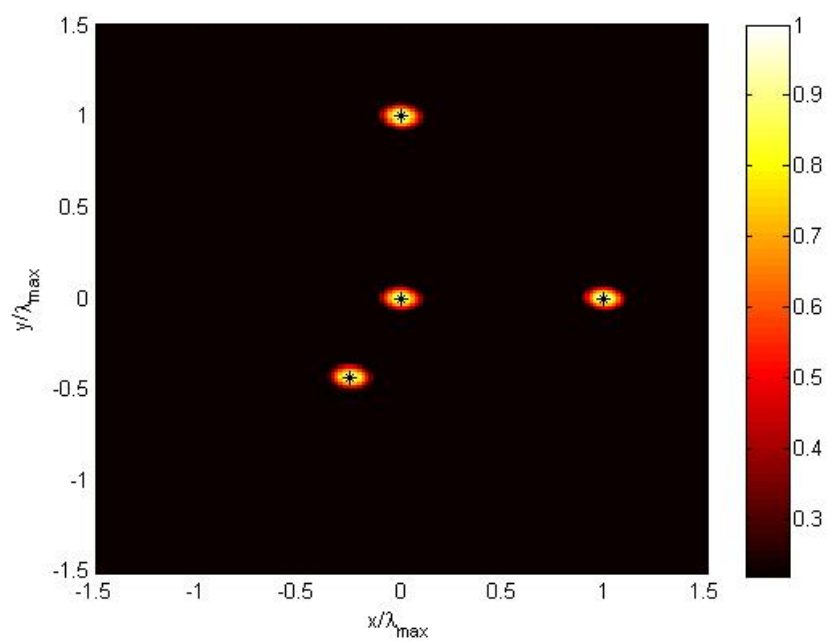

Fig. 2. Reconstructed positions of four cylinders of radius $a=0.1 \lambda_{\text {max }}$ (the actual positions are indicted by a black star).

\section{Discussion}

Let us analyze the importance of a correct knowledge of the radius $a$ in order to localize the scatterers.

Actually, the assumed value of $a$ influences the normalization factor $F(\mathrm{ka})$, whose expression is given in Eq. (6). In the present multibistatic configuration $\left(\theta-\theta_{i}\right)$ has a constant known value and the normalization factor depends only on $k a$, i.e. on the scatterers dimension with respect to the wavelength.

The function $F(k a)$ is nothing else that the scattered field by a single circular cylinder along the $\theta$ direction for plane wave incidence from direction $\theta_{i}$. As well known (Tyras, 1969) the Watson transform and asymptotic arguments allow to approximate such a factor as the Geometric Optics reflected field so that it is proportional to $-E_{0} \sqrt{a / 2 * \cos (\Delta \theta / 2)} \exp [+j 2 k a \cos (\Delta \theta / 2)-$ $j k r]$ where $\Delta \theta=\theta-\theta_{i}-\pi$ is the offset between the observation and the incidence direction. Therefore, the amplitude of $F(k a)$ is constant and the phase shows a linear frequency behavior. While the influence of the phase function is discussed in detail hereafter, the independence of the modulus of $F(k a)$ from the frequency makes the SVD decomposition of the full operator (5) equal to the one considered in (Pierri et al., 2005) with the same singular values behavior.

Once the normalization has been performed, if the radius value is not exactly known, a residue phase factor $\exp (j k \Delta r)$ is present in the operator linking data and unknown (note that $\Delta r$ can be both positive and negative). In this case (see Appendix A) the integral kernel in Eq. (5) can be written as:

$e^{j k \Delta r} e^{-j(u x+v y)}=e^{-j[u(x+\Delta x)+v(y+\Delta y)]}$

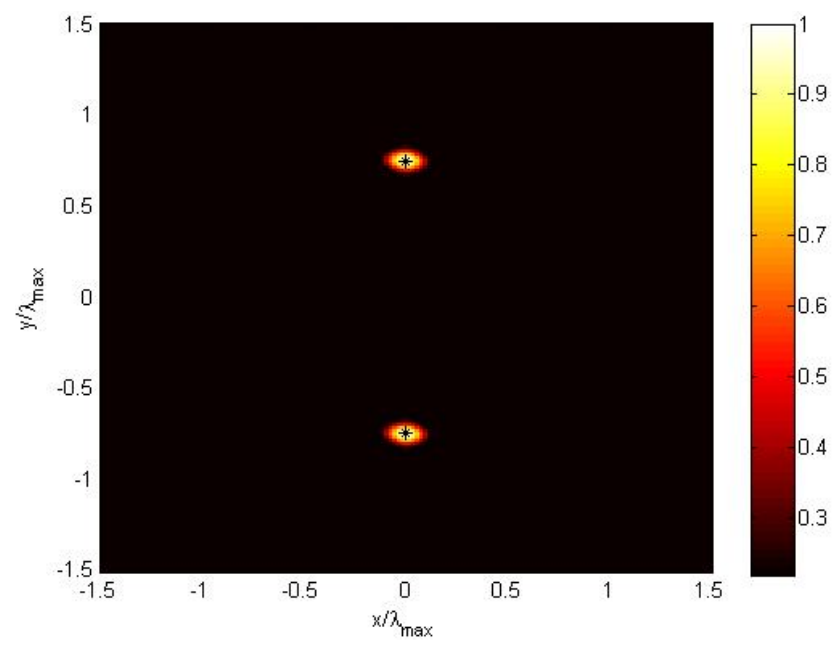

Fig. 3. Reconstructed positions of two cylinders of radius $a=0.5 \lambda_{\text {max }}$ (the actual positions are indicted by a black star).

where

$\Delta x=-\frac{\Delta r}{2(1+\cos \Delta \theta)}\left[(1+\cos \Delta \theta) \cos \theta_{i}-\sin \Delta \theta \sin \theta_{i}\right]$

and

$\Delta y=-\frac{\Delta r}{2(1+\cos \Delta \theta)}\left[(1+\cos \Delta \theta) \sin \theta_{i}+\sin \Delta \theta \cos \theta_{i}\right]$

Therefore such an error affects the localization of the cylinder by an amount connected to $\Delta x$ and $\Delta y$. In Appendix A we show that, for each scatterer, instead of a peaked function in the reconstructed image, an arc of circle of radius $|\Delta r| / \sqrt{2(1+\cos \Delta \theta)}$, centered on the actual scatterer position, should be expected. In particular, if the radius $a$ is underestimated $\Delta r>0$ and the reconstructed image appears as an arc convex with respect to the measurement domain; instead, in case of overestimation $\Delta r<0$ and the reconstructed image appears as an arc concave with respect to the measurement domain.

\section{Numerical results}

We consider a realistic geometry in which data are collected in a reflection mode under a multi-bistatic configuration.

A stepped frequency illumination is considered, so that $k \in\left[\frac{2 \pi f_{\min } \sqrt{\epsilon_{r}}}{c_{0}}, \frac{2 \pi f_{\max } \sqrt{\epsilon_{r}}}{c_{0}}\right]$ where $f_{\max }$ and $f_{\min }$ are the maximum and minimum frequency respectively, and $c_{0}$ is the speed of light in a vacuum space. In the following examples we use $f_{\max }=2 \mathrm{GHz}, f_{\min }=400 \mathrm{MHz}$, with a frequency step of $50 \mathrm{Mhz}, \epsilon_{r}=4$. The investigation domain is $D=\left[-1.5 \lambda_{\max }, 1.5 \lambda_{\max }\right] \times\left[-1.5 \lambda_{\max }, 1.5 \lambda_{\max }\right]$ in the $x, y$ plane, where $\lambda_{\max }$ is the maximum wavelength calculated inside the background medium $\left(\lambda_{\max }=37.5 \mathrm{~cm}\right.$ in the examples at hand). The measurements are taken over an arc of 


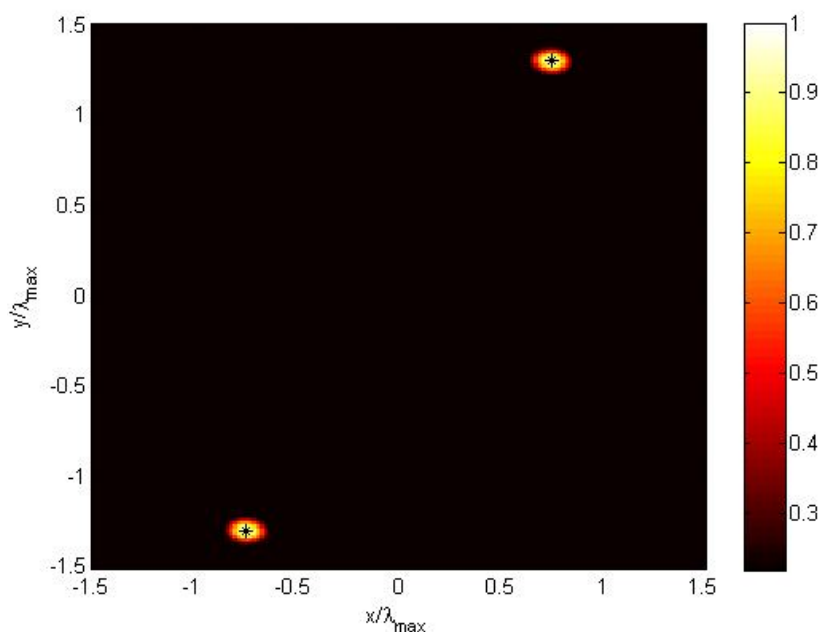

Fig. 4. Reconstructed positions of two cylinders of radius $a=1.5 \lambda_{\max }$ (the actual positions are indicted by a black star).

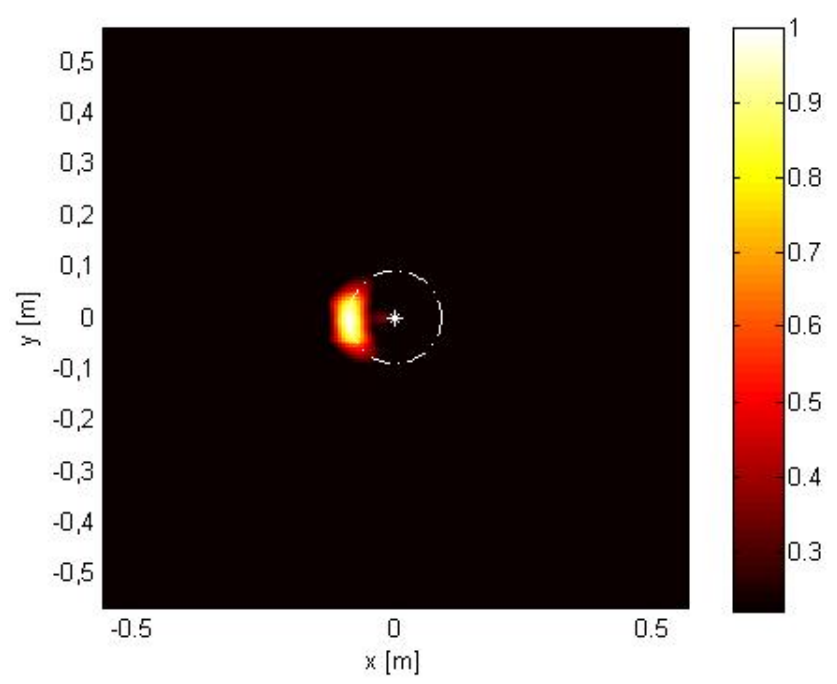

Fig. 5. Reconstruction result for a cylinder of radius $a=10 \mathrm{~cm}$ when a wrong value $a=1 \mathrm{~cm}$ is assumed in the inversion algorithm (the actual position is indicted by a white star).

$\pi / 2 \mathrm{rad}$ and the offset between the source and the receiver is $\pi / 20 \mathrm{rad}$, i.e. $\theta-\theta_{i}=\pi-\pi / 20 \mathrm{rad}$.

In all the following examples, the SVD is truncated to the singular value index $10 \mathrm{~dB}$ lower than the maximum one. Moreover, the reconstructed functions are normalized to their maximum value and the final images are not depicted under a threshold level 0.21 times the maximum one.

First, in order to show the effectiveness of the proposed algorithm, we assume that the radius $a$ of the cylinders is known exactly. In Figs. (2), (3), (4) the reconstructed positions of cylinders of radius $a=0.1 \lambda_{\max }, a=0.5 \lambda_{\max }$, $a=1.5 \lambda_{\max }$ are shown respectively. As can be seen, the ac-

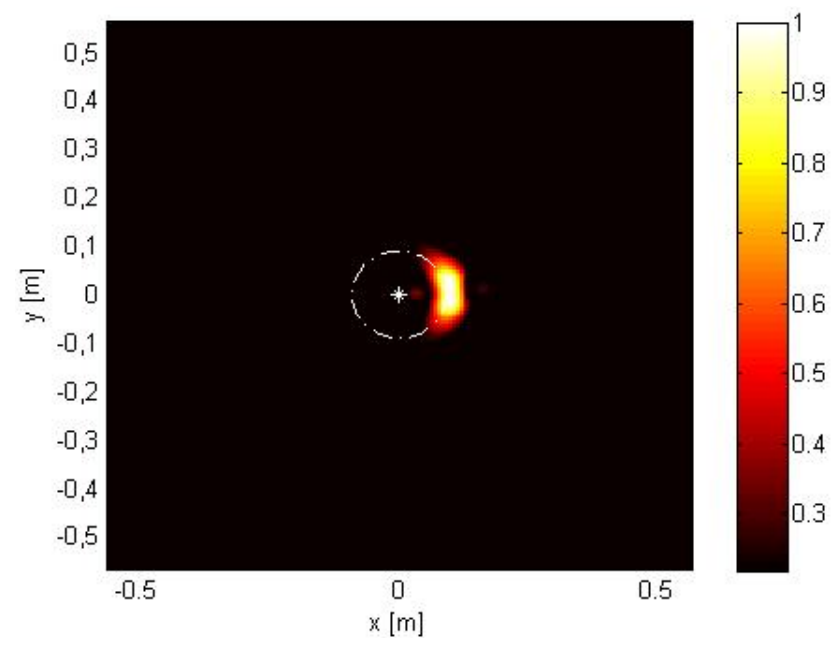

Fig. 6. Reconstruction result for a cylinder of radius $a=10 \mathrm{~cm}$ when a wrong value $a=20 \mathrm{~cm}$ is assumed in the inversion algorithm (the actual position is indicted by a white star).

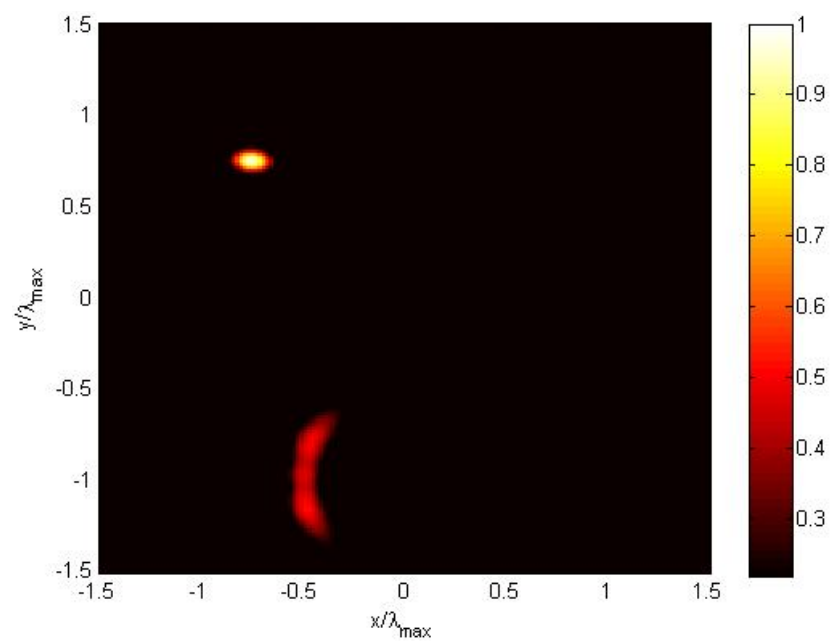

Fig. 7. Reconstruction result for two cylinders of radius $a=0.7 \lambda_{\max }$ and $a=1.2 \lambda_{\max }$, located at $(x, y)=\left(-0.75 \lambda_{\max }, 0.75 \lambda_{\max }\right)$ and $(x, y)=\left(0,-1.2 \lambda_{\max }\right)$ respectively; $a=0.7 \lambda_{\max }$ is assumed in the inversion algorithm.

tual positions (indicated by a black star) are well recovered.

As an example of the influence of the correct knowledge of the cyilinders' radius, discussed in the section above, the reconstruction relative to a cylinder of radius $a=10 \mathrm{~cm} \mathrm{ob}-$ tained by assuming a lower $a=1 \mathrm{~cm}$ and a higher $a=20 \mathrm{~cm}$ values are shown in Figs. (5) and (6) respectively. As it has been anticipated, the reconstruction is an arc convex with respect to the measurement domain when the radius is underestimated, concave in the opposite case. 
We consider now the case in which the scatterers have different radii. In this case, the algorithm can provide the correct localization of the cylinders whose radius is equal to that used in the normalization factor $F(k a)$, while, following the arguments discussed above, we expect that the position of any other cylinder should be reconstructed as a circular arc. Such a result is confirmed by the example shown in Fig. 7.

\section{Conclusions}

In this paper we have performed the extension of an algorithm for localization of thin PEC cylinders to a collection of identical cylinders of arbitrary radius. The algorithm has been validated against simulated data and the effect of errors in the knowledge of the cylinders' radius on the reconstructed images has been examined.

We considered the case of far zone scattered field in reflection mode configuration and limited extension of the observation domain, that is preliminary to subsurface prospection. The extension to dielectric objects, near zone observation and half space geometry is under development.

\section{Appendix A}

Let us define the unitary vectors: $\hat{i}_{\theta_{i}}=\left(\cos \theta_{i}, \sin \theta_{i}\right)$, indicating the incident field propagation direction, $\hat{i}_{\perp}=\left(\sin \theta_{i},-\cos \theta_{i}\right)$, orthogonal to $\hat{i}_{\theta_{i}}$ in the $x, y$ plane, and the vector $\underline{i}_{D}=(1+\cos \Delta \theta,-\sin \Delta \theta)$ (where the $x$ and $y$ cartesian components are indicated in brackets). We can express the variables $u$ and $v$ (introduced in Eq. 5) as a function of $\Delta \theta$ :

$u=k(1+\cos \Delta \theta) \cos \theta_{i}-k \sin \Delta \theta \sin \theta_{i}$,

$v=k(1+\cos \Delta \theta) \sin \theta_{i}+k \sin \Delta \theta \cos \theta_{i}$,

that can be rewritten, by exploiting the above defined vectors, as:

$u=k \underline{i}_{D} \cdot \hat{i}_{\theta_{i}} ; v=k \underline{i}_{D} \cdot \hat{i}_{\perp}$,

Let us now write the product $k \Delta r$ as a function of the vector $\underline{i}_{D}$ :

$k \Delta r=\frac{k \underline{i}_{D} \cdot \Delta r \underline{i}_{D}}{\left|\underline{i}_{D}\right|^{2}}$

Then, we can project each vector at the second member of Eq. A4 onto $\hat{i}_{\theta_{i}}$ and $\hat{i}_{\perp}$. So, we have:

$$
\begin{aligned}
& \left(k \underline{i}_{D} \cdot \hat{i}_{\theta_{i}} \hat{i}_{\theta_{i}}+k \underline{i}_{D} \cdot \hat{i}_{\perp} \hat{i}_{\perp}\right) \cdot\left(\Delta r \underline{i}_{D} \cdot \hat{i}_{\theta_{i}} \hat{i}_{\theta_{i}}+\Delta r \underline{i}_{D} \cdot \hat{i}_{\perp} \hat{i}_{\perp}\right)= \\
& =u \Delta r \underline{i}_{D} \cdot \hat{i}_{\theta_{i}}+v \Delta r \underline{i}_{D} \cdot \hat{i}_{\perp}
\end{aligned}
$$

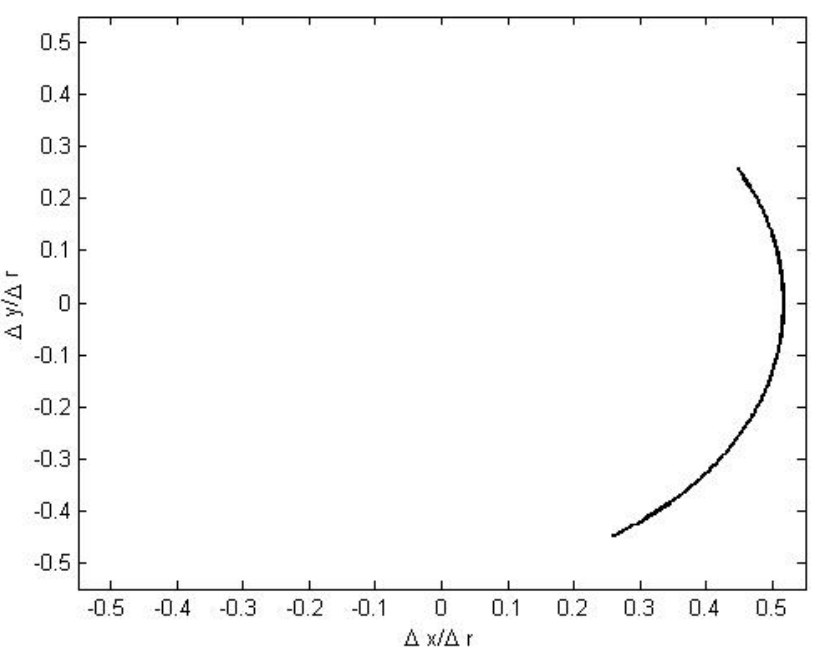

Fig. A1. Expected displacement in the reconstruction in presence of a phase error.

where the expressions Eq. A3 for $u$ and $v$ have been used. Finally:

$k \Delta r-u x-v y=-u\left(x-\frac{\Delta r \underline{i}_{D} \cdot \hat{i}_{\theta_{i}}}{2(1+\cos \Delta \theta)}\right)-$

$-v\left(y-\frac{\Delta r \underline{i}_{D} \cdot \hat{i}_{\perp}}{2(1+\cos \Delta \theta)}\right)$.

We can conclude that, in presence of a phase factor $k \Delta r$, a displacement with respect to the coordinates $x, y$ is experienced. Such displacement is given by

$$
\begin{aligned}
& \Delta x=-\frac{\Delta r}{2(1+\cos \Delta \theta)} \underline{i}_{D} \cdot \hat{i}_{\theta_{i}} \\
& =-\frac{\Delta r}{2(1+\cos \Delta \theta)}\left[(1+\cos \Delta \theta) \cos \theta_{i}-\sin \Delta \theta \sin \theta_{i}\right]
\end{aligned}
$$

and

$$
\begin{aligned}
& \Delta y=-\frac{\Delta r}{2(1+\cos \Delta \theta)} \underline{i}_{D} \cdot \hat{i}_{\perp} \\
& =-\frac{\Delta r}{2(1+\cos \Delta \theta)}\left[(1+\cos \Delta \theta) \sin \theta_{i}+\sin \Delta \theta \cos \theta_{i}\right]
\end{aligned}
$$

That are the expressions reported in Eqs. 8 and 9. Such displacements represent the parametric equation of a circle of radius $|\Delta r| / \sqrt{2(1+\cos \Delta \theta)}$ in the $x, y$ plane, at variance of the incidence angle $\theta_{i}$. In Fig. A1 an example of such a curve is plotted for $\Delta \theta=\pi / 6$ and $\theta_{i} \in[-\pi / 4, \pi / 4]$, in the case $\Delta r>0$.

Edited by: F. Soldovieri

Reviewed by: two anonymous referees 


\section{References}

Pierri, R., Solimene, R., Liseno, A., and Romano, J.: Linear distribution imaging of thin metallic cylinders under mutual scattering, IEEE Trans. Antennas Propag., 53, 3019-3029, 2005.

Soldovieri, F., Brancaccio, A., Leone, G., and Pierri, R.: Shape Reconstruction of Perfectly Conducting Objects by Multiview Experimental Data, IEEE Trans. Geosc. Remote Sens., 43, 65-71, 2005.

Harrington, R. F.: Time Harmonic Electromagnetic Fields, Ed. Wiley and Sons, 2001.
Brancaccio, A., Leone, G., and Pierri, R.: Information content of Born scattered fields: results in the circular cylindrical case, J. Opt. Soc. Am., 15, 1909-1917, 1998.

Liseno, A., Soldovieri, F., and Pierri, R.: Improving a shape reconstruction algorithm with thresholds and multi-view data, AEU, International Journal of Electronics and Communications, 58, 118-124, 2004.

Soldovieri, F. and Persico, R.: Reconstruction of an Embedded Slab from Multifrequency Scattered Field Data Under the Distorted Born Approximation, IEEE Trans. Antennas Propag., 52, 23482356, 2004.

Tyras G.: Radiation and propagation of electromagnetic waves, Academic Press, New York, 1969 